\title{
Genetic diversity of Plasmodium falciparum AMA-1 antigen from the Northeast Indian state of Tripura and comparison with global sequences: implications for vaccine development
}

Tulika Nirmolia ${ }^{1 \dagger}$, Md. Atique Ahmed ${ }^{1}$, Vinayagam Sathishkumar ${ }^{1}$, Nilanju P. Sarma ${ }^{1,6}$, Dibya R. Bhattacharyya', Pradyumna K. Mohapatra', Devendra Bansal ${ }^{2,5}$, Praveen K. Bharti ${ }^{3}$, Rakesh Sehgal', Jagadish Mahanta', Ali A. Sultan ${ }^{2}$, Kanwar Narain ${ }^{1}$ and Saurav J. Patgiri ${ }^{1{ }^{*+}}$ (1)

\begin{abstract}
Background: Malaria continues to be a major public health problem in the Northeastern part of India despite the implementation of vector control measures and changes in drug policies. To develop successful vaccines against malaria, it is important to assess the diversity of vaccine candidate antigens in field isolates. This study was done to assess the diversity of Plasmodium falciparum AMA-1 vaccine candidate antigen in a malaria-endemic region of Tripura in Northeast India and compare it with previously reported global isolates with a view to assess the feasibility of developing a universal vaccine based on this antigen.
\end{abstract}

Methods: Patients with fever and malaria-like illness were screened for malaria and P. falciparum positive cases were recruited for the current study. The diversity of PfAMA-1 vaccine candidate antigen was evaluated by nested PCR and RFLP. A selected number of samples were sequenced using the Sanger technique.

Results: Among 56 P. falciparum positive isolates, Pfama- 1 was successfully amplified in $75 \%(n=42)$ isolates. Allele frequencies of PfAMA-1 antigen were 16.6\% $(n=7)$ for 3D7 allele and $33.3 \%(n=14)$ in both K1 and HB3 alleles. DNA sequencing revealed 13 haplotypes in the Pfama-1 gene including three unique haplotypes not reported earlier. No unique amino-acid substitutions were found. Global analysis with 2761 sequences revealed 435 haplotypes with a very complex network composition and few clusters. Nucleotide diversity for Tripura $(0.02582 \pm 0.00160)$ showed concordance with South-East Asian isolates while recombination parameter $(\mathrm{Rm}=8)$ was lower than previous reports from India. Population genetic structure showed moderate differentiation.

Conclusions: Besides documenting all previously reported allelic forms of the vaccine candidate PfAMA-1 antigen of P. falciparum, new haplotypes not reported earlier, were found in Tripura. Neutrality tests indicate that the Pfama-1 population in Tripura is under balancing selection. This is consistent with global patterns. However, the high

\footnotetext{
*Correspondence: saurav.patgiri@gmail.com

${ }^{\dagger}$ Tulika Nirmolia and Saurav J. Patgiri contributed equally as first authors

${ }^{1}$ ICMR - Regional Medical Research Centre, North East Region, Dibrugarh, Assam 786001, India

Full list of author information is available at the end of the article
}

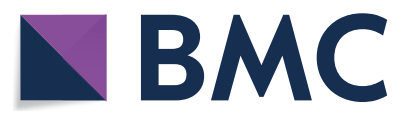

(c) The Author(s) 2022. Open Access This article is licensed under a Creative Commons Attribution 4.0 International License, which permits use, sharing, adaptation, distribution and reproduction in any medium or format, as long as you give appropriate credit to the original author(s) and the source, provide a link to the Creative Commons licence, and indicate if changes were made. The images or other third party material in this article are included in the article's Creative Commons licence, unless indicated otherwise in a credit line to the material. If material is not included in the article's Creative Commons licence and your intended use is not permitted by statutory regulation or exceeds the permitted use, you will need to obtain permission directly from the copyright holder. To view a copy of this licence, visit http://creativecommons.org/licenses/by/4.0/. The Creative Commons Public Domain Dedication waiver (http://creativeco mmons.org/publicdomain/zero/1.0/) applies to the data made available in this article, unless otherwise stated in a credit line to the data. 
haplotype diversity observed in the global Pfama-1 network analysis indicates that designing a universal vaccine based on this antigen may be difficult. This information adds to the existing database of genetic diversity of field isolates of $P$. falciparum and may be helpful in the development of more effective vaccines against the parasite.

Keywords: Apical Membrane Antigen 1 (AMA-1), Plasmodium falciparum, Genetic diversity, Tripura, Northeast India

\section{Background}

The World Health Organization (WHO) estimates approximately 229 million new malaria cases and 409,000 deaths due to malaria occurring globally in 2019 [1]. Out of this, South-East Asia contributes 3\% of the total caseload. In 2019, India's contribution to total malaria cases and deaths in the South-East Asian region was around $88 \%$ and $86 \%$ respectively. In South-East Asia, Plasmodium vivax is now the major (51.7\%) malaria parasite in circulation [1].

On an average, North East India contributes approximately 7\% of the total malaria cases in India [2]. Among the eight states of North East India, Tripura is highly malaria endemic where transmission is persistent [3]. Many outbreaks of malaria have been reported in the past few decades from Tripura [4]. In 2014, a severe malaria outbreak occurred in the state with a high morbidity and mortality rate reported mainly from the Dhalai district $[5,6]$.

Despite active vector control strategies and artemisinin-based combination therapy (ACT) being implemented universally, drug resistant and genetically diverse Plasmodium falciparum is spreading continuously across different parts of the world including North East India [7-10]. An effective vaccine is needed, but despite many efforts initiated throughout the last six decades, still no licensed vaccine is available which shows $100 \%$ efficacy against the disease. Recently the RTS,S/AS01 vaccine has gone through phase-III efficacy trial which and has shown partial protection (30-50\%) against P. falciparum malaria in young children [11-15]. RTS,S/AS01 is the only first-generation malaria vaccine and its largescale pilot implementation has started in April, 2019 in Malawi, Ghana and Kenya [16, 17]. Based on these results, in October 6, 2021 the World Health Organization recommended the widespread use of RTS,S/AS01 malaria vaccine among children living in sub-Saharan Africa and other regions with moderate to high P. falciparum malaria transmission [18].

The main obstacle in producing an effective malaria vaccine is the highly polymorphic nature of the parasite and vaccine candidate genes, which allows the parasite to escape host immunity [19]. The erythrocytic stage of the malaria parasite is important in this respect because most vaccine candidate proteins such as the merozoite surface protein $(M S P)$ are expressed while invading the RBCs coinciding with clinical disease [20]. However, these vaccine candidate proteins are highly polymorphic in nature, mandating a detailed understanding of their diversity patterns through field studies in different geographical locations $[21,22]$. Among the established vaccine candidate antigens of $P$. falciparum, blood-stage antigens like MSPs have traditionally been given more importance; but other antigens like $P$. falciparum apical membrane antigen (PfAMA-1) also hold promise [23].

One of the leading erythrocytic stage vaccine candidate genes of $P$. falciparum is apical membrane antigen-1 (AMA-1). It is $83 \mathrm{kDa}$ in size and expressed in the late schizont stage of the life cycle of malaria parasite [24]. As described previously, this gene can be classified into three major allelic families by PCR-RFLP technique based on the amino acid differences present outside the Hyper Variable Region (HVR) of the gene [25]. Although its function is not yet clear, many studies documented that antibodies against PfAMA-1 raised in rabbits can inhibit the invasion of red blood cells by both homologous and heterologous $P$. falciparum and vaccines based on PfAMA-1 can induce asymptomatic protection [26-29]. Despite its less polymorphic nature, antibodies to this antigen have shown to confer natural protection against the disease [30-32]. Phase-I vaccine trial has also been conducted on malaria-naive volunteers [33]. It was also reported by previous studies that immunization with PfAMA-1 provides protection against malaria in mice and monkeys [34, 35].

Malaria control requires a coordinated approach based on vector control strategies and basic research such as surveillance of parasite genetic diversity and evolution. Very few studies have reported the diversity of Pfama-1 gene in northeastern as well as in other parts of India [36-39]. The current study was thus conducted to address this void and to analyse the diversity patterns between Tripura and other parts of the world with a view to understand whether a universal vaccine based on this candidate would be feasible in the near future.

\section{Methods \\ Study location}

This study was carried out in the sub-divisional hospitals, primary health centers and villages of Dhalai $\left(23.8467^{\circ} \mathrm{N}\right.$, $\left.91.9099^{\circ} \mathrm{E}\right)$ and North Tripura $\left(24.0797^{\circ} \mathrm{N}, 92.2630^{\circ} \mathrm{E}\right)$ districts of Tripura state, India in 2015. These districts 
share borders with Bangladesh in the north and south and more than $70 \%$ of the land area is covered by hills and forests. The area experiences hot, humid summers and a prolonged rainy season.

\section{Study population}

Symptomatic patients with body temperature $\geq 37.5^{\circ} \mathrm{C}$, age $>1$ year, without history of anti-malarial drug consumption and no recent history of fever were included in this study. Presence of $P$. falciparum parasite was screened by rapid diagnostic test (RDT) and confirmed by slide microscopy. The study was conducted with approval from the Institutional Human Ethics committee of ICMR-RMRC North East Region (No. RMRC/DIB./ IEC Human/ 2012/667) and all protocols were carried out as per the guidelines of the Indian Council of Medical Research (ICMR). Two $\mathrm{ml}$ of whole blood was collected from the $P$. falciparum positive patients after obtaining informed written consent.

\section{Genomic DNA isolation and Plasmodium species identification}

Genomic DNA extraction was done from whole blood samples using QIAamp DNA blood mini kit as per the manufacturer's protocol (Qiagen, CA, USA). Plasmodium species specific nested PCR was carried out for confirmation of $P$. falciparum as described previously [40]. Extracted DNA was stored in $-20^{\circ} \mathrm{C}$ for further molecular analysis.

\section{Nested PCR for Pfama-1 gene}

All the PCR protocols and primers were used as previously described with minor modifications [41]. Primary PCR for Pfama-1 was performed using $1 \mu \mathrm{l}$ of genomic DNA and $1 \mu \mathrm{M}$ of forward and reverse primers in a $10 \mu \mathrm{l}$ reaction volume containing $5 \mu \mathrm{l}$ of $2 \mathrm{X}$ Promega master mix. Secondary PCR was performed using $2 \mu \mathrm{l}$ of primary PCR product as template with $1 \mu \mathrm{M}$ of each primer and $25 \mu \mathrm{l}$ of $2 \mathrm{X}$ Promega master mix in $50 \mu \mathrm{l}$ reaction volumes. The PCR amplification conditions were: initial denaturation at $95{ }^{\circ} \mathrm{C}$ for $5 \mathrm{~min}$ followed by (30 cycles for primary PCR and 35 cycles for secondary PCR) denaturation at $95{ }^{\circ} \mathrm{C}$ for $2 \mathrm{~min}$, annealing at $52{ }^{\circ} \mathrm{C}$ for $30 \mathrm{~s}$, extension at $68^{\circ} \mathrm{C}$ for $45 \mathrm{~s}$ and a final extension of $5 \mathrm{~min}$ at $68{ }^{\circ} \mathrm{C}$. The PCR product of Pfama-1 was analysed on $2 \%$ agarose gel and expected positive amplicon size was $500 \mathrm{bp}$.

\section{Restriction fragment length polymorphism analysis of Pfama-1}

After successful amplification of the Pfama-1 gene, the amplified PCR products were subjected to digestion with three restriction enzymes, viz. Mse1, Ssp1 and Sau3A1
(New England Biolabs) as previously described [42]. Three independent digestions were performed with the three restriction enzymes. The digestion mixture contained $0.4 \mu \mathrm{l}$ restriction enzyme, $2 \mu \mathrm{l} 10 \mathrm{X}$ buffer, $10 \mu \mathrm{l}$ PCR product and volume made up to $20 \mu \mathrm{l}$ by adding nuclease free water. Digestion was done for $60 \mathrm{~min}$ at $37^{\circ} \mathrm{C}$ followed by 20 min enzyme inactivation at $65^{\circ} \mathrm{C}$ as described by manufacturer's protocol with minor modifications. The digested products were analysed on $2.5 \%$ agarose gel. The respective band sizes for Mse1, Ssp1 and Sau3A1 enzyme digestions were 285 bp (K1), 400 bp (3d7) and 335 bp (HB3), respectively.

\section{Sequencing}

A limited number of samples (17 out of 42 isolates with successful Pfama-1 amplification) were selected for sanger sequencing. Samples for sequencing were selected randomly since all the 42 samples which showed positive amplification for Pfama-1 also showed successful restriction digestion with each of the three enzymes. Since the Indian and global database on Pfama-1 is quite extensive, sequencing was done for a small number of samples representative of the geographical region under consideration. Selected samples were gel purified using Wizard ${ }^{\circledR}$ SV Gel and PCR Cleanup System (Promega) following manufactures's protocol. The purified products were outsourced to Eurofins Genomic India private limited, Banglore, for both forward and reverse direction Sanger sequencing.

\section{Sequence polymorphism, phylogenetic and statistical analysis}

The sequences were edited in the software Bioedit v7.0.5.3 and aligned in Clustal W [43, 44]. The BLAST similarity searches were done in GenBank database and representative sequences from other parts of the world were downloaded for comparison. The Plasmodium reichenowi strain (Accession No. AJ252087) was included as an outgroup for performing the neutrality tests i.e. Fu \& Li's F and D test. DnaSP version 6 was used to calculate various measures of genetic polymorphism such as haplotype diversity $(\mathrm{Hd})$, nucleotide diversity $(\pi)$, recombination parameters $(R)$ and different neutrality tests [45]. The $\pi$ value was calculated to estimate step-wise diversity based on a sliding window of 100 bases with a step size of $25 \mathrm{bp}$. The genetic differentiation among the populations based on fixation index (Fst) was estimated by using Arlequin 3.5 software [46]. Taking $P$. reichenowi strain as an outgroup, $\mathrm{dN} / \mathrm{dS}$ values were estimated using SNAP v2.1.1 [47]. Haplotype network of global Pfama-1 haplotypes was constructed with 2761 sequences following the Minimum Spanning Network algorithm using the software PopART [48]. The list of sequences downloaded 
from the NCBI database and used in the population genetics and Network analysis is provided as a separate file (Additional file 1: Table S1).

\section{Results}

A total of 56 P. falciparum PCR positive isolates were included in this study for genetic diversity analysis. Among these, Pfama-1 was amplified in $75.0 \%(\mathrm{n}=42)$ isolates.

\section{Allele prevalence among the isolates}

In positive samples, all the three previously reported alleles were observed with fragment sizes of $285 \mathrm{bp}$ (K1), $400 \mathrm{bp}$ (3d7) and $335 \mathrm{bp}$ (HB3) after restriction digestion.

Table 1 Allele frequency of Pfama-1 gene in Tripura

\begin{tabular}{lll}
\hline Total sample [56] & Allele & Frequency \\
\hline & K1 & $33.30 \%(n=14)$ \\
& $3 D 7$ & $16.60 \%(n=7)$ \\
Pfama-1 $(n=42)$ & HB3 & $33.30 \%(n=14)$ \\
& K1+3D7+HB3 & $2.40 \%(n=1)$ \\
K1+3D7 & $2.40 \%(n=1)$ \\
3D7+HB3 & $4.70 \%(n=2)$ \\
HB3+K1 & $4.70 \%(n=2)$ \\
\hline
\end{tabular}

Among the 42 positive isolates, frequencies of $\mathrm{K} 1$ and HB3 allele types were $33.3 \%(n=14)$ each and 3D7 allele type was $16.6 \%(n=7)$, respectively (Table 1$)$. Besides the three allele types, mixed alleles were also observed in three isolates. An additional allele fragment, not previously reported, bearing a size of approximately $355 \mathrm{bp}$ was observed in $83.3 \%(n=35)$ isolates.

\section{DNA sequencing and sequence polymorphisms}

Pfama-1 gene was successfully sequenced for a total of 17 $P$. falciparum isolates. The sequences were submitted to the NCBI database with Accession numbers: MT483644 to MT483628. In this study, a total number of 13 different haplotypes were observed in Pfama-1 antigenic gene of Tripura P. falciparum isolates (Table 2). Out of these, three unique haplotypes were observed: H-3/MT483630, H6-/MT483634, MT483637 and H-9/MT483638 when compared with 2744 global Pfama-1 sequences. Further, it was observed that the H-5/MT483633 haplotype was reported only from India, the H-8/MT483636 haplotype was reported earlier from India and Myanmar and the H-7/MT483635 haplotype was reported only from Uganda. Other eight haplotypes were earlier reported from various parts of the world (Table 2). On multiple sequence alignment of the 17 Pfama-1 nucleotide sequences with the reference strain (XM-001347979), 21 amino acid substitutions were found in domain 1 of

Table 2 Pfama-1 haplotypes observed in the study and their geographical distribution

\begin{tabular}{|c|c|c|c|c|c|}
\hline Gene & $\begin{array}{l}\text { No. of seq. } \\
\text { analysed }\end{array}$ & $\begin{array}{l}\text { No. of } \\
\text { haplotype }\end{array}$ & $\begin{array}{l}\text { Haplotype } \\
\text { (H) }\end{array}$ & $\begin{array}{l}\text { Accession No. } \\
\text { (Sample ID) }\end{array}$ & Reported from \\
\hline & & & $\mathrm{H}-1$ & $\begin{array}{l}\text { MT483628 } \\
\& \\
\text { MT483631 }\end{array}$ & $\begin{array}{l}\text { India, Benin: Cotonou, Ghana, Tanzania, Philippines, Gambia, Mali, Uganda, Kenya, } \\
\text { Nigeria, Saudi Arabia }\end{array}$ \\
\hline \multirow[t]{12}{*}{ Pfama-1 } & 17 & 13 & $\mathrm{H}-2$ & MT483629 & Uganda, Ghana, Gambia, Mali, Kenya, Nigeria, Saudi Arabia \\
\hline & & & $\mathrm{H}-3$ & MT483630 & This study \\
\hline & & & $\mathrm{H}-4$ & MT483632 & India, Iran, Thailand, Myanmar, Tanzania, Kenya \\
\hline & & & $\mathrm{H}-5$ & MT483633 & India \\
\hline & & & $\mathrm{H}-6$ & $\begin{array}{l}\text { MT483634 } \\
\& \\
\text { MT383637 }\end{array}$ & This study \\
\hline & & & $\mathrm{H}-7$ & MT483635 & Uganda \\
\hline & & & $\mathrm{H}-8$ & MT483636 & India, Myanmar \\
\hline & & & $\mathrm{H}-9$ & MT483638 & This study \\
\hline & & & $\mathrm{H}-10$ & $\begin{array}{l}\text { MT483639 } \\
\& \\
\text { MT483641 }\end{array}$ & India, Kenya, Nigeria, Mali, Gambia, Cameroon \\
\hline & & & $\mathrm{H}-11$ & MT383640 & Thailand, Malaysia, Philippines, Solomon Island, Papua New Guinea \\
\hline & & & $\mathrm{H}-12$ & MT483642 & $\begin{array}{l}\text { India, Myanmar, Thailand, Solomon Island, Philippines, Kenya, Mali, Tanzania," } \\
\text { Gambia, Papua New Guinea, Saudi Arabia, Uganda }\end{array}$ \\
\hline & & & $\mathrm{H}-13$ & $\begin{array}{l}\text { MT483643 } \\
\& \\
\text { MT483644 }\end{array}$ & India, Myanmar, Thailand, China, Mali, Vanuatu, Papua New Guinea \\
\hline
\end{tabular}


Pfama-1 gene (Fig. 1). Eighteen dimorphic (N162K, T167K, G172E, N173K, Y175D, L189P, M190I, D196N, F201L, D204N, K206E, Y207D, I225N, N228K, K230E, D242Y, K245N, E267Q), two trimorphic (H200D/L, K243N/E) and two tetramorphic (E187N/D/K, E197G/ $\mathrm{D} / \mathrm{Q})$ substitutions were found.

\section{Haplotype network analysis}

Global analysis with 2761 sequences (including 17 sequences from current study and one reference $3 \mathrm{~d} 7$ strain) from NCBI database revealed 435 haplotypes out of which $261(60 \%)$ were singletons. Three haplotypes detected in the current study were unique (Hap_299/ MT483630, Hap_301_/MT483634, MT483637 and Hap_302/MT483638) and not found amongst the analysed global sequences (Fig. 2). The analysis included sequences from Tripura and 23 malaria endemic countries, including India. From the haplotype network, it was observed that although there was clustering of haplotypes from different countries, the overall picture was complex. Indian isolates in particular appeared to be clustered with a number of global isolates; however, it was observed that there were many unique haplotypes from India not reported earlier from other parts of the world. As many as 169 haplotypes were obtained from the 266 Indian sequences (excluding those from Tripura in the present study) included in the analysis. This was followed by Mali, Kenya and Gambia with 109, 59 and
48 haplotypes each. Three major haplotypes were seen in the Minimum Spanning Network analysis: Hap_1 (176 isolates including the 3d7 reference strain), Hap_3 (161 isolates) and Hap_188 (135 isolates). Hap_1 contained isolates from 15 countries spread across Africa, South America, Oceania and South-East Asia including India; the maximum number of isolates were from Mali (30.68\%). Hap_3 included isolates from the Middle East, South and South-East Asia (including Tripura), Africa and Oceania; highest frequency of isolates belonged to Myanmar (49.07\%). Hap_188 isolates predominantly belonged to Middle East, South and South-East Asia and Oceania, with no African isolates.

\section{Nucleotide diversity and natural selection of Pfama-1 isolates from Tripura compared to global isolates}

For analysis of nucleotide diversity and natural selection of Pfama-1 isolates, the sequences from Tripura were compared with other Indian isolates $(\mathrm{n}=266)$ as well as isolates $(n=2485)$ from twenty other malaria endemic countries spread out across Asia, Middle-East, South America, Africa and Oceania. For the 17 Tripura P. falciparum sequences included in this analysis, the calculated nucleotide diversity $(\pi)$ was 0.02582 and average number of pairwise nucleotide differences $(\mathrm{k})$ was 10.610 . When these values were compared with other global $P$. falciparum isolates, it was observed that $\pi$ value was similar to South-East Asian isolates (Thailand $=0.02565$,

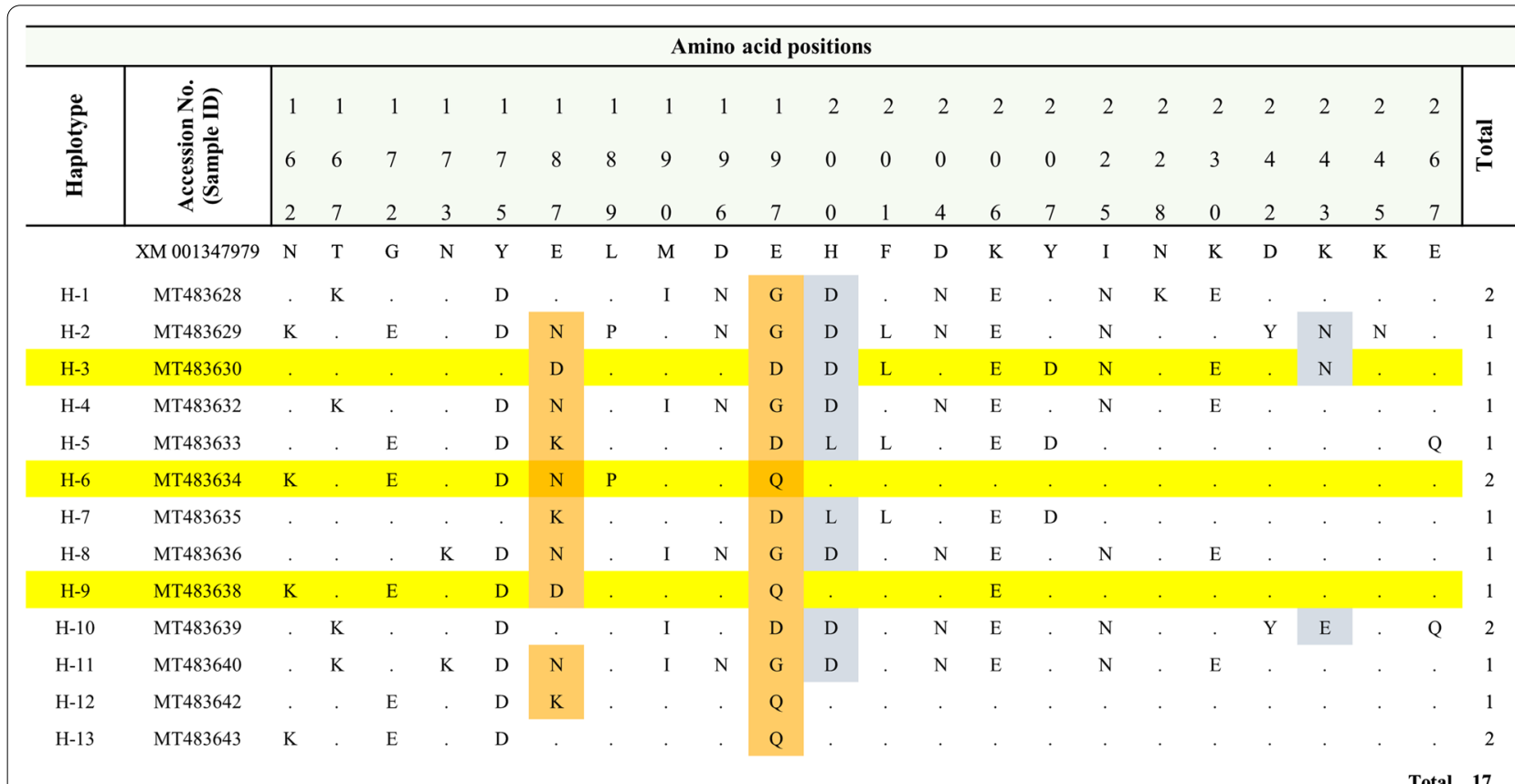

Fig. 1 Amino-acid sequence polymorphism observed in Tripura Pfama-1 isolates. Trimorphic and tetramorphic amino acid are highlighted in blue and orange respectively. New haplotype $(\mathrm{H}-3, \mathrm{H}-6$ and $\mathrm{H}-9)$ are highlighted in yellow 


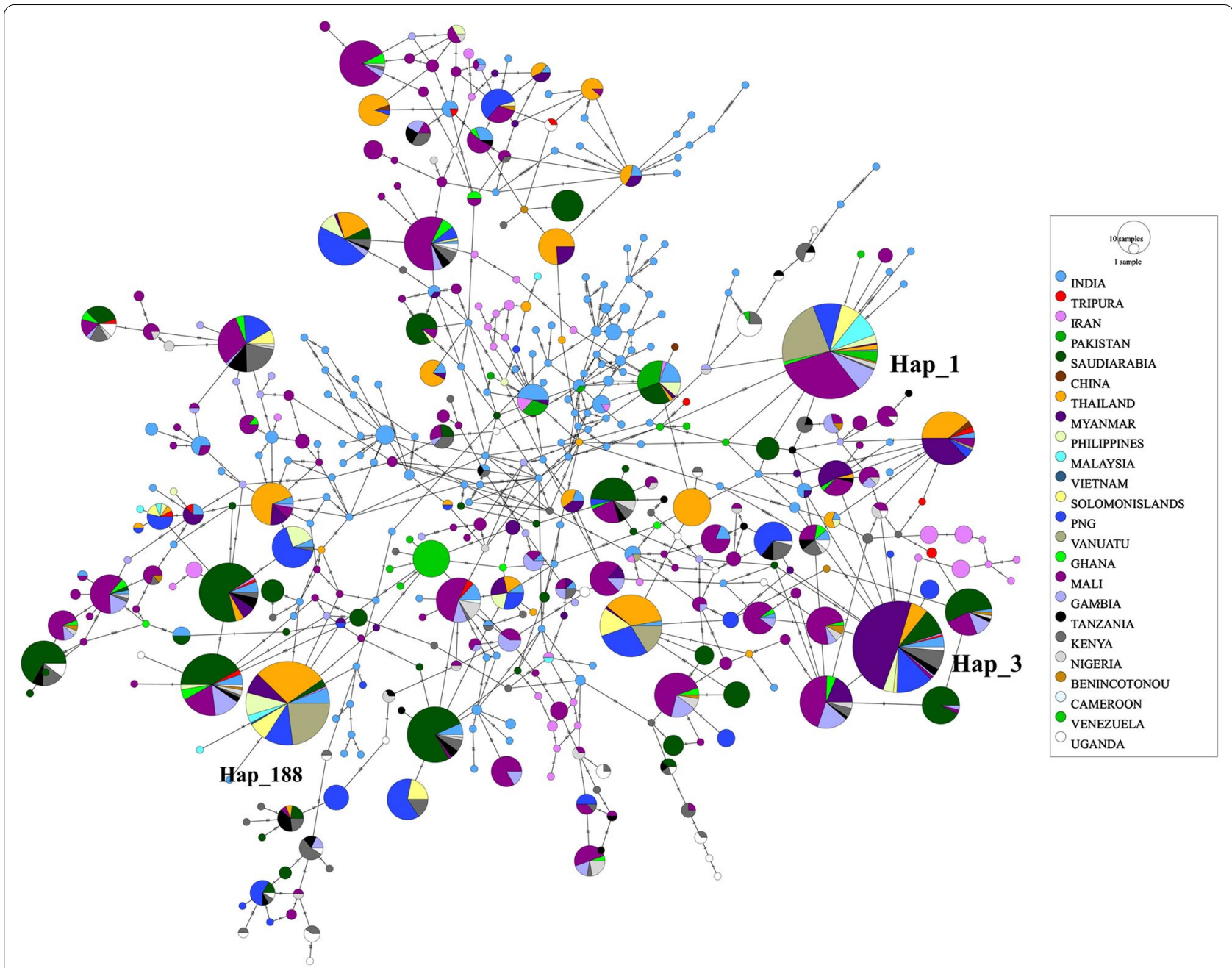

Fig. 2 Haplotype network of global Pfama-1 isolates created using PopArt (Minimum Spanning Network algorithm). Isolates are colour-coded according to the country of origin and the size of the vertex represents the frequency of the haplotype. Hap_1, Hap_3 and Hap_188 are marked separately. Haplotypes from Tripura observed in this study are shown in red

Philippines $=0.02512$ ). Highest nuclotide diversity was observed in Uganda $(\pi=0.03187)$ and lowest was observed among South American (Venezuela $=0.01469)$ and South-East Asian (Pakistan $=0.01348)$ isolates. The average number of pairwise nucleotide differences (k) in the isolates included in the analysis ranged from 5.464 in Venezuela to 11.124 in Kenya (Table 3). The Tripura isolates $(k=10.610)$ had values similar to other Indian isolates $(\mathrm{k}=10.847)$ and isolates from Middle East (Saudi Arabia $=10.570$ ) and Africa (Papua New Guinea $=10.158$, Ghana $=10.991$, Mali $=10.409$, Nigeria $=10.475$, Benin:Cotonou $=10.773$, Gambia $=10.455$, Tanzania $=10.731$ ). The neutrality tests in Pfama-1 gene i.e. Fu and Li's D \& $\mathrm{F}$ test were performed considering $P$. reichenowi as an outgroup species. For the Tripura isolates, the observed values for Fu and Li's D \& F test were
1.36492 and 1.58826, respectively, which were not statistically significant (Table 3). Positive Tajima's D value (1.14014, $\mathrm{P}>0.10$ ), though statistically not significant and a positive $\mathrm{dN} / \mathrm{dS}$ ratio (2.2237), indicated that the Pfama-1 gene of Tripura P. falciparum isolates was under positive selection indicating that there was no evidence of population bottle-necking. However, inclusion of greater number of sequences from Tripura would have increased the accuaracy of these neutrality tests. When possible, Tajima's D value was calculated for the other global $P$. falciparum isolates and it was found that it was positive for all countries except India $(-1.64633,0.10>\mathrm{P}>0.05)$ and China $(-0.01883, \mathrm{P}>0.10)$ (Table 3$)$. This signifies that these isolates may be under negative selection which differs from other isolates of the world that show balancing or positive selection. The negative values as extracted by Fu and Li's D and F test also supported the negative 


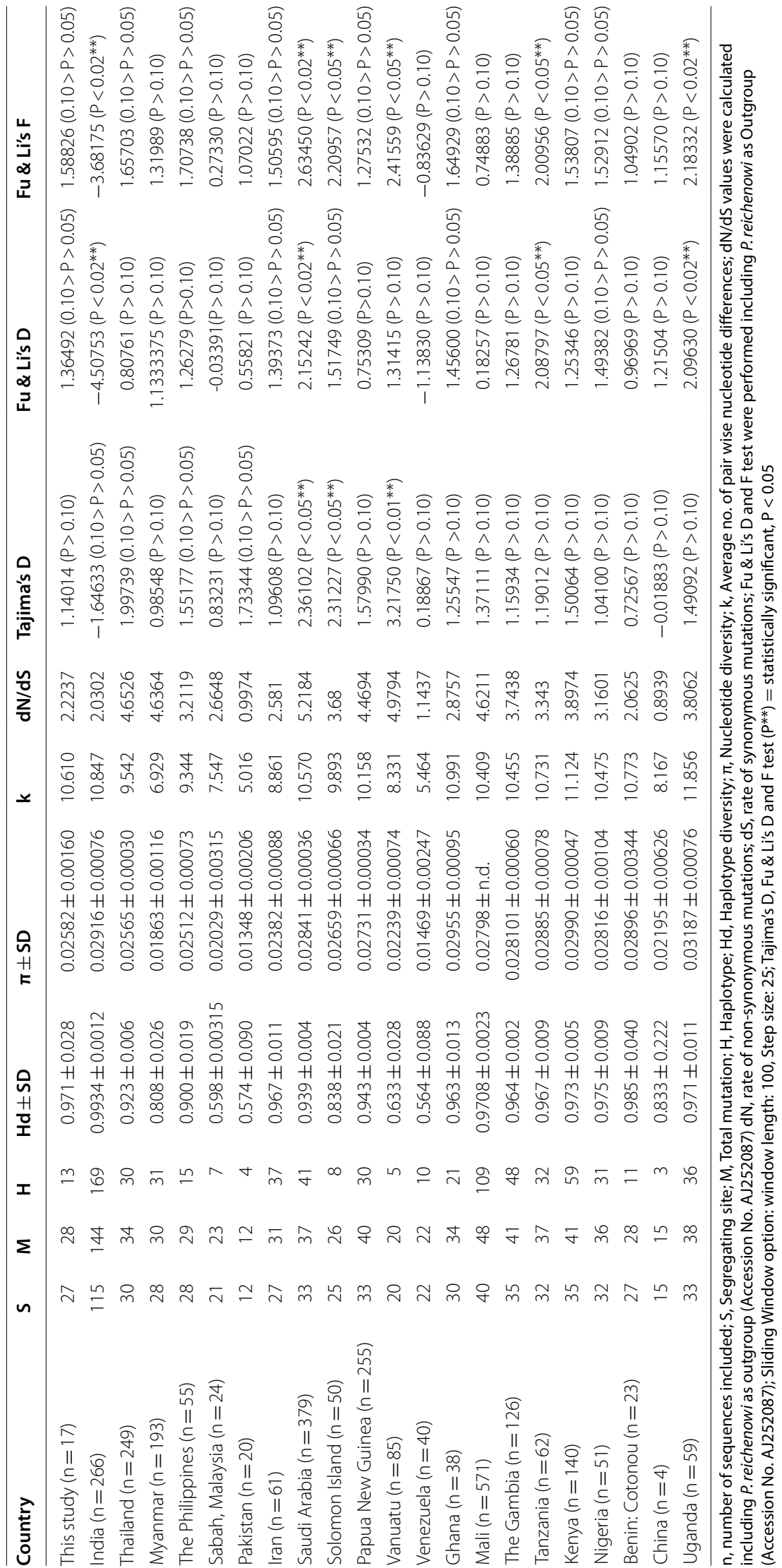


selection pattern for Indian isolates and the values were statistically significant.

\section{Recombination parameter}

The minimum number of recombination events ( $\mathrm{Rm}$ ) was calculated along with $\mathrm{R}$ values of adjacent sites ( $R a)$ and per gene $(R b)$. The estimated $R m$ and $R$ values observed in this study were $8(\mathrm{Rm}), 0.0954(\mathrm{Ra})$ and 39.1 $(\mathrm{Rb})$, respectively. When compared with other global $R m$ and $R$ values, it was observed that the $R m$ value of the present study was similar to South-East Asia (Thailand $=8$, Myanmar =8) and African countries (Benin: Cotonou $=8$ ). However, previously reported Pfama1 isolates from India showed the highest $\mathrm{Rm}$ values $(R m=24)$, which is in contrast to this study. Similarly, Ra values observed in this study were similar to those from Saudi Arabia, Papua New Guinea, Ghana and Tanzania. Highest Ra values were observed in India, Nigeria and Uganda. $\mathrm{Rb}$ values from Tripura showed similarity with isolates from Iran and the Philippines. Highest $\mathrm{Rb}$ values amongst the analysed samples were seen from African countries (Ghana, Mali, Gambia, Tanzania, Kenya, Nigeria and Benin: Cotonou) (Table 4).

\section{Inter-population differentiation}

Fst values were evaluated to estimate the genetic differentiation among global Pfama-1 populations (Additional file 2: Table S2). Among all the global isolates, lowest Fst value was observed between Kenya and Uganda (0.00479, $\mathrm{p}<0.05)$ and highest Fst value was observed between China and Venezuela $(0.44783, \mathrm{p}=0)$. When Fst values were compared for Tripura isolates, lowest difference was with Tanzania $(0.01168, \mathrm{p}>0.05)$ and highest difference was with Venezuela $(0.24724, p=0)$. Low genetic difference of Tripura isolates was also observed with the earlier reported Indian isolates as well as other South-East Asian countries like Thailand, Malaysia, Philippines; African countries like Ghana, Uganda, Benin, Gambia, Kenya and other countries like Saudi Arabia, Mali and PNG, etc. Moderate genetic difference was seen with the isolates of Iran, China and Myanmar. Highest genetic difference was seen with the isolates of Pakistan and Venezuela. However, there was one negative Fst value which was seen between the isolates of Kenya and Tanzania $(-0.00052$, $\mathrm{p}>0.05$ ); this may be due to geographical proximity or inclusion of short sequences in the analysis.

\section{Discussion}

The WHO South-East Asia region comprises of eleven countries and is home to more than a quarter of the world's population. Nine of these eleven countries are endemic for malaria with three countries, including India, contributing to over $99.5 \%$ of the total caseload
Table 4 Recombination events of Pfama-1 gene among global P. falciparum isolates

\begin{tabular}{lcll}
\hline Country & Rm & Ra & Rb \\
\hline This study & 8 & 0.0954 & 39.1 \\
India & 24 & 0.1864 & 79.8 \\
Thailand & 8 & 0.0445 & 57.9 \\
Myanmar & 8 & 0.0074 & 10 \\
The Philippines & 6 & 0.0218 & 40.8 \\
Sabah, Malaysia & 5 & 0.0001 & 0.2 \\
Pakistan & 0 & 0 & 0.001 \\
Iran & 12 & 0.074 & 39 \\
Saudi Arabia & 13 & 0.0857 & 54 \\
Solomon Island & 5 & 0.012 & 22.5 \\
Papua New Guinea & 9 & 0.1094 & 63 \\
Vanuatu & 5 & 0.001 & 1.8 \\
Venezuela & 1 & 0 & 0.001 \\
Ghana & 10 & 0.0958 & 179 \\
Mali & 12 & 0.1131 & 137 \\
The Gambia & 12 & 0.082 & 146 \\
Tanzania & 9 & 0.0915 & 171 \\
Kenya & 13 & 0.1171 & 153 \\
Nigeria & 10 & 0.158 & 207 \\
Benin: Cotonou & 8 & 0.0755 & 119 \\
China & 0 & 0.0581 & 29.3 \\
Uganda & 12 & 0.1822 & 67.6 \\
\hline Rn, & & &
\end{tabular}

$\mathrm{Rm}$, Minimum number of recombination events between adjacent sites; $\mathrm{Ra}$, Recombination parameter between adjacent sites; Rb, Recombination parameter per gene

in the region [1]. In India, there are several hotspots of malaria across the country with diverse epidemiological, ecological and geographical settings. Vector and parasite species also vary depending on the region. Northeast India is a very peculiar setting wherein forest malaria predominates; the region also shares a huge international border and has traditionally acted as a gateway for the spread of drug resistant Plasmodium strains from surrounding countries to the Indian mainland [4, 49, 50]. Although malaria has reduced drastically in this region over the years, further reduction and control has been difficult. Anti-malarial drug resistance monitoring and surveillance of parasite genetic diversity and evolution are important molecular tools to understand and minimize the spread of malaria in this region. Currently, Tripura and Mizoram are the two states from this region reporting a substantial number of malaria cases [2]. Many areas in Tripura are endemic for malaria and not much data on diversity of $P$. falciparum vaccine candidate genes like Pfama-1 is available on field isolates. The current study was done to assess the diversity of Pfama-1 population circulating in the region through RFLP and Sanger sequencing 
with a view to compare it with other Indian and global isolates.

Restriction fragment length polymorphism (RFLP) analysis of Pfama-1 gene showed the presence of all the three reported allelic variants; i.e. K1, 3D7 and HB3 type in the study population with a frequency of $33.30 \%$, $16.60 \%$ and $33.30 \%$ respectively. In addition to this, $2.4 \%$ mixed allele type was observed among the $P$. falciparum isolates and $83.3 \%$ (355 bp) isolates did not fit into any of these previously reported groups. Earlier studies among Indian $P$. falciparum isolates have reported mostly point mutations; suggesting that PCR-RFLP based genotyping for Pfama-1 allele needs further validation since it is inherently incapable of detecting SNPs [37]. The current study has shown a lower frequency of the 3D7 allele as compared to $\mathrm{K} 1$ and $\mathrm{HB} 3$ allelic variants, which is similar to previous studies from South-East Iran, East Africa, Western and Central Africa [30, 41, 51]. However, many other studies based on SNPs have reported varying frequencies of Pfama-1 haplotype in different geographical regions [22, 52-54]. Kang et al. also reported close similarities between the Pfama-1 gene in Thailand and Myanmar [53]. Polymorphism in Pfama-1 gene is not evenly distributed; antigenic diversity of Pfama-1 between and among global isolates are limited and also it is relatively less polymorphic in nature than the laboratory isolates, possibly due to the positive natural selection and genetic recombination $[30,37,53,54]$. In high disease transmission areas, allelic diversity of Pfama-1 is usually very high compared to low transmission areas and in major endemic areas, Pfama-1 alleles have been found to exhibit similar diversity patterns regardless of geographical region $[39,52,55]$.

Sanger sequencing of a few Pfama-1 isolates revealed thirteen haplotypes in total out of which three haplotypes were unique as depicted in the haplotype network constructed using 2761 global Pfama-1 sequences (Fig. 2). The haplotype network was dense with many complex connections and no clear clustering was apparent. As many as 435 haplotypes were identified out of which $60 \%$ were singletons; India being a major contributor to these low frequency haplotypes. There was no haplotype that was present universally in all the malaria endemic countries included in the analysis. Three haplotypes with sizeable number of isolates from different countries were obtained out of which Hap_1, which included the $P$. falciparum 3D7 strain, formed the biggest cluster with 176 isolates (6.37\% of analysed strains). Most of the PfAMA-1 antigen-based vaccines that are currently being evaluated for field use have been designed on the basis of $P$. falciparum 3D7 strain. The current analysis revealed that only a minority of the global isolates belonged to this haplotype (Hap_1); additionally, only one isolate from
India and none from Tripura were identical to this haplotype. Studies conducted in Myanmar and Bioko islands have also observed similar haplotype networks with no consistent clustering; although, the number of isolates included in the network analysis was much smaller [53, 56]. In Myanmar, haplotype network constructed using 517 global Pfama-1 isolates revealed 174 haplotypes and in a separate study conducted in Bioko islands, 296 haplotypes were obtained from the analysis of 790 sequences $[53,56]$.

Nucleotide diversity figures observed in this study ( $\pi=0.02582 \pm 0.00160$ ) were found to be similar to South-East Asian and African isolates and slightly lower than those reported earlier from India (Table 3). However, studies conducted in nearby Indian states like Assam and Orissa have reported similar values [36]. Even Andaman \& Nicobar Islands, an Indian union territory and archipelago in the Bay of Bengal, has reported values for nucleotide diversity $(0.0226 \pm 0.0008)$ similar to the Tripura isolates [36]. Another study from India has also reported similar nucleotide diversity patterns from Assam, Orissa and North India [39]. Haplotype diversity and average number of pairwise nucleotide differences (k) were also found to be similar in Tripura, Assam, Orissa and Andaman \& Nicobar Islands [36]. Haplotype diversity of domain-I of Tripura Pfama-1 strains was found to be higher than that reported earlier from SouthEast Asian countries such as Myanmar and Thailand and comparable to those observed in African countries like Ghana and Tanzania $[53,56]$. A positive Tajima's D value for majority of global isolates (except India and China, Table 3) also indicates that the domain-1 of Pfama-1 is under positive natural selection; this has been reported earlier in several studies $[36,53,56]$. Negative values for Tajima's D, which signify negative selection, have been reported earlier from India in Kolkata [39]. A possible explanation for this may be the high number of low frequency haplotypes (as many as $60 \%$ of the Indian haplotypes were singletons) observed in the 266 Pfama-1 sequences included in the analysis. Nucleotide diversity, haplotype diversity and recombination events $(R m)$ were also highest among the Indian isolates $(\pi=0.02916 \pm$ $0.00076, H d=0.9934 \pm 0.0012, R m=24)$, which signifies that the parasite population must have undergone recent expansion with recombination events generating newer alleles.

In the current study, no unique amino-acid substitutions were observed; all amino-acid substitutions reported here have been observed previously in Tanzania, Ghana, Thailand, Bioko island, Pakistan and some other parts of the world $[53,56,57]$. Of these, the N228K mutation encountered in the current study has been found to be more common in African countries unlike 
Myanmar and Thailand [53]. Although there are differences in the frequency of these amino-acid substitutions across different geographical regions, the overall distribution is largely the same; however, inclusion of larger number of samples from diverse geographical regions of North East India would have provided a more comprehensive picture.

Genetic differentiation among global Pfama-1 populations was evaluated by estimating the pairwise Fst values and classified as previously described [53]. Tripura isolates exhibited low levels of genetic differentiation when compared to African (Uganda, Tanzania, Nigeria, Mali, Kenya, Ghana, Gambia and Benin: Cotonou) countries. Moderate levels of differentiation were observed with previously reported Indian isolates (0.05351) and isolates from South-East Asia (Myanmar, Malaysia), Iran, China and Vanuatu. High-level differentiation was observed when the Tripura isolates were compared with those of Venezuela (0.24724) and Pakistan (0.22606). This trend is more or less similar to that shown by other Indian isolates when compared globally: high differentiation with Venezuela (0.16519) and Iran (0.15053); moderate differentiation with Vanuatu, Tanzania, Solomon Islands, Pakistan, Myanmar, Malaysia, China and Benin: Cotonou; and low differentiation with all other countries (predominantly African) included in the analysis. Interestingly, it was observed that isolates from Venezuela, the only South American country included in the analysis, showed a high level of genetic differentiation with all other countries. This might be due to the geographical barriers to gene flow among the parasite populations of Venezuela and the other countries (which belong to different continents) analysed. A similar finding is also observed in a study conducted in Bioko Islands where the Venezuelan strains showed high genetic differentiation with nine countries from Africa, South-East Asia and Oceania [56]. Other studies have found moderate levels of differentiation (0.05-0.15) among global Pfama-1 sequences [53]. Differences in the number of sequences and the country of origin analysed may sometimes lead to variations in the absolute Fst values; however, the overall picture for Pfama-1 appears to be one of low to moderate genetic differentiation with a few exceptions, which can be explained based on the isolation by distance model. Overall, nucleotide and haplotype diversity figures, haplotype network and inter-population Fst values indicate that a common vaccine design may be complicated.

Limitations of the current study include sequencing of a limited number of samples for the target gene. Additionally, the samples were subjected to both way Sanger sequencing without cloning. While most traditional studies on Pfama-1 have used PCR-RFLP, we have also used Sanger sequencing, which is one of the strengths of this study. Moreover, no previous study has compared the global Pfama-1 scenario on such a huge scale.

\section{Conclusions}

The study aimed to evaluate the diversity of PfAMA1 , a less commonly studied vaccine candidate antigen of $P$. falciparum from Tripura, a malaria endemic state in Northeast India. All the three allelic families were observed and neutrality tests indicate that the Pfama-1 population in Tripura is under balancing selection. This is consistent with global patterns. However, the high haplotype diversity observed in the global Pfama-1 network analysis indicates that designing a universal vaccine based on this antigen may be difficult. A moderate degree of clustering of isolates from different geographical regions indicate that region specific vaccines based on the PfAMA-1 antigen may have some hope for malaria control.

\section{Abbreviations}

AMA-1: Apical Membrane Antigen 1; PCR: Polymerase chain reaction; RFLP Restriction fragment length polymorphism; WHO: World Health Organization; NVBDCP: National Vector Borne Disease Control Programme; ACT: Artemisininbased combination therapy; RDT: Rapid diagnostic test.

\section{Supplementary Information}

The online version contains supplementary material available at https://doi. org/10.1186/s12936-022-04081-1.

Additional file 1: List of Sequences used in phylogenetic and Haplotype network analysis of Pfama-1 gene.

Additional file 2: Pair wise Fst values of global Pfama-1 gene.

\section{Acknowledgements}

The authors acknowledge the Indian Council of Medical Research for providing infrastructure, laboratory and logistical support. The authors are thankful to the study participants, Tripura health government authorities and the entire staff of Malaria Division, ICMR-RMRC N. E. Region, Dibrugarh for their support.

\section{Authors' contributions}

SP, NS, PM., DB, PB, RS, JM and AS designed the experiments. SP, NS, TN, VS, AA, performed the experiments. SP, NS, PM., DB, DRB, PB and KN analysed the data. TN, SP, VS, AA drafted the manuscript. All the authors read and approved the final manuscript.

\section{Funding}

The Qatar National Research Fund (QNRF) through Grant No. NPRP 5-098-3021 funded the study.

\section{Data availability}

All sequences generated in this study are available in the NCBI database (https://www.ncbi.nlm.nih.gov/) and with the corresponding author. The list of additional sequences used in the analysis is available in the Supplementary materials.

\section{Declarations}

Ethics approval and consnet to partciicpate

The study was conducted with approval from the Institutional Human Ethics committee of ICMR-RMRC North East Region (No. RMRC/DIB./IEC Human/ 
2012/ 667) and all protocols were carried out as per the guidelines of the Indian Council of Medical Research.

\section{Consent for publication}

Not applicable.

\section{Competing interests}

The authors declare no conflict of interest.

\section{Author details}

${ }^{1}$ ICMR - Regional Medical Research Centre, North East Region, Dibrugarh, Assam 786001, India. ${ }^{2}$ Department of Microbiology and Immunology, Weill Cornell Medicine - Qatar, Cornell University, Doha, Qatar. ${ }^{3}$ ICMR - National Institute for Research in Tribal Health, Jabalpur, Madhya Pradesh 482003, India. ${ }^{4}$ Department of Medical Parasitology, Postgraduate Institute of Medical Education and Research, Chandigarh, Punjab 160012, India. ${ }^{5}$ Present Address: Ministry of Public Health, Doha, Qatar. ${ }^{6}$ Present Address: SRL Reference Laboratory, Mumbai 400060, India.

Received: 10 September 2021 Accepted: 7 February 2022 Published online: 22 February 2022

\section{References}

1. WHO. World malaria report 2020: 20 years of global progress and challenges. Geneva, World Health Organization; 2020.

2. National Vector Borne Disease Control Programme. Malaria Situation in India from 2016. MoHFW, Delhi, India.

3. Shah NK, Dhillon GP, Dash AP, Arora U, Meshnick SR, Valecha N. Antimalarial drug resistance of Plasmodium falciparum in India: changes over time and space. Lancet Infect Dis. 2011;11:57-64.

4. Dhiman S, Gopalakrishnan R, Goswami D, Rabha B, Baruah I, Singh L. Malaria incidence among paramilitary personnel in an endemic area of Tripura. Indian J Med Res. 2011;133:665-9.

5. Bhattacharjee U. More than 100 die as Tripura battles malaria. NDTV.com. 2014.

6. Sarmah NP, Bhowmik IP, Sarma DK, Sharma CK, Medhi GK, Mohapatra PK, et al. Role of Anopheles baimaii: potential vector of epidemic outbreak in Tripura, North-east India. J Glob Health Rep. 2019;3:e2019036.

7. Mishra N, Singh JP, Srivastava B, Arora U, Shah NK, Ghosh SK, et al. Monitoring antimalarial drug resistance in India via sentinel sites: outcomes and risk factors for treatment failure, 2009-2010. Bull World Health Organ. 2012;90:895-904.

8. Phyo AP, Nkhoma S, Stepniewska K, Ashley EA, Nair S, McGready R, et al. Emergence of artemisinin-resistant malaria on the western border of Thailand: a longitudinal study. Lancet. 2012;379:1960-6.

9. Kyaw MP, Nyunt MH, Chit K, Aye MM, Aye KH, Lindegardh N, et al. Reduced susceptibility of Plasmodium falciparum to artesunate in southern Myanmar. PLoS ONE. 2013:8:e57689.

10. Mishra N, Prajapati SK, Kaitholia K, Bharti RS, Srivastava B, Phookan S, et al. Surveillance of artemisinin resistance in Plasmodium falciparum in India using the kelch13 molecular marker. Antimicrob Agents Chemother. 2015;59:2548-53.

11. Bejon P, Lusingu J, Olotu A, Leach A, Lievens M, Vekemans J, et al. Efficacy of RTS,S/ASO1E vaccine against malaria in children 5 to 17 months of age. N Engl J Med. 2008;359:2521-32.

12. Guinovart C, Aponte JJ, Sacarlal J, Aide P, Leach A, Bassat Q, et al. Insights into long-lasting protection induced by RTS,S/AS02A malaria vaccine: further results from a phase Ilb trial in Mozambican children. PLoS ONE. 2009;4:e5165.

13. Aide P, Aponte JJ, Renom M, Nhampossa T, Sacarlal J, Mandomando I, et al. Safety, immunogenicity and duration of protection of the RTS,S/ AS02(D) malaria vaccine: one year follow-up of a randomized controlled phase I/IIb trial. PLOS ONE. 2010;5:e13838.

14. Hill AVS. Vaccines against malaria. Philos Trans R Soc Lond Ser B Biol Sci. 2011;366:2806-14.

15. Olotu A, Lusingu J, Leach A, Lievens M, Vekemans J, Msham S, et al. Efficacy of RTS,S/AS01E malaria vaccine and exploratory analysis on anti-circumsporozoite antibody titres and protection in children aged 5-17 months in Kenya and Tanzania: a randomised controlled trial. Lancet Infect Dis. 2011;11:102-9.

16. Schuerman L. RTS,S malaria vaccine could provide major public health benefits. Lancet. 2019;394:735-6.

17. Adepoju P. RTS,S malaria vaccine pilots in three African countries. Lancet. 2019;393:1685.

18. WHO recommends groundbreaking. malaria vaccine for children at risk. Geneva: World Health Organization; 2021.

19. Riley EM, Stewart VA. Immune mechanisms in malaria: new insights in vaccine development. Nat Med. 2013;19:168-78.

20. Tolia NH, Enemark EJ, Sim BK, Joshua-Tor L. Structural basis for the EBA175 erythrocyte invasion pathway of the malaria parasite Plasmodium falciparum. Cell. 2005;122:183-93.

21. Mu J, Awadalla P, Duan J, McGee KM, Keebler J, Seydel K, et al. Genome-wide variation and identification of vaccine targets in the Plasmodium falciparum genome. Nat Genet. 2007;39:126-30.

22. Mardani A, Keshavarz H, Heidari A, Hajjaran H, Raeisi A, Khorramizadeh MR. Genetic diversity and natural selection at the domain I of apical membrane antigen-1 (AMA-1) of Plasmodium falciparum in isolates from Iran. Exp Parasitol. 2012;130:456-62

23. Sawaswong V, Simpalipan P, Siripoon N, Harnyuttanakorn P, Pattaradilokrat S. Allelic diversity and geographical distribution of the gene encoding Plasmodium falciparum Merozoite Surface Protein-3 in Thailand. Korean J Parasitol. 2015;53:177-87.

24. Peterson MG, Marshall VM, Smythe JA, Crewther PE, Lew A, Silva A, et al. Integral membrane protein located in the apical complex of Plasmodium falciparum. Mol Cell Biol. 1989;9:3151-4.

25. Marshall VM, Zhang L, Anders RF, Coppel RL. Diversity of the vaccine candidate AMA-1 of Plasmodium falciparum. Mol Biochem Parasitol. 1996:77:109-13.

26. Stowers AW, Kennedy MC, Keegan BP, Saul A, Long CA, Miller LH. Vaccination of monkeys with recombinant Plasmodium falciparum apical membrane antigen 1 confers protection against blood-stage malaria. Infect Immun. 2002;70:6961-7.

27. Kocken $\mathrm{CH}$, Withers-Martinez $\mathrm{C}$, Dubbeld MA, van der Wel A, Hackett F, Valderrama A, et al. High-level expression of the malaria blood-stage vaccine candidate Plasmodium falciparum apical membrane antigen 1 and induction of antibodies that inhibit erythrocyte invasion. Infect Immun. 2002;70:4471-6.

28. Nair M, Hinds MG, Coley AM, Hodder AN, Foley M, Anders RF, et al. Structure of domain III of the blood-stage malaria vaccine candidate, Plasmodium falciparum apical membrane antigen 1 (AMA1). J Mol Biol. 2002;322:741-53.

29. Silvie O, Franetich JF, Charrin S, Mueller MS, Siau A, Bodescot M, et al. A role for apical membrane antigen 1 during invasion of hepatocytes by Plasmodium falciparum sporozoites. J Biol Chem. 2004:279:9490-6.

30. Escalante AA, Grebert HM, Chaiyaroj SC, Magris M, Biswas S, Nahlen $\mathrm{BL}$, et al. Polymorphism in the gene encoding the apical membrane antigen-1 (AMA-1) of Plasmodium falciparum. X. Asembo Bay Cohort Project. Mol Biochem Parasitol. 2001;113:279-87.

31. Cortes A, Mellombo M, Mueller I, Benet A, Reeder JC, Anders RF. Geographical structure of diversity and differences between symptomatic and asymptomatic infections for Plasmodium falciparum vaccine candidate AMA1. Infect Immun. 2003;71:1416-26.

32. Udhayakumar V, Kariuki S, Kolczack M, Girma M, Roberts JM, Oloo AJ, et al. Longitudinal study of natural immune responses to the Plasmodium falciparum apical membrane antigen (AMA-1) in a holoendemic region of malaria in western Kenya: Asembo Bay Cohort Project VIII. Am J Trop Med Hyg. 2001;65:100-7.

33. Malkin EM, Diemert DJ, McArthur JH, Perreault JR, Miles AP, Giersing BK, et al. Phase 1 clinical trial of apical membrane antigen 1: an asexual blood-stage vaccine for Plasmodium falciparum malaria. Infect Immun. 2005:73:3677-85.

34. Deans JA, Knight AM, Jean WC, Waters AP, Cohen S, Mitchell GH. Vaccination trials in rhesus monkeys with a minor, invariant, Plasmodium knowlesi 66 kD merozoite antigen. Parasite Immunol. 1988;10:535-52.

35. Anders RF, Crewther PE, Edwards S, Margetts M, Matthew ML, Pollock $B$, et al. Immunisation with recombinant AMA-1 protects mice against infection with Plasmodium chabaudi. Vaccine. 1998;16:240-7. 
36. Garg S, Alam MT, Das MK, Dev V, Kumar A, Dash AP, et al. Sequence diversity and natural selection at domain I of the apical membrane antigen 1 among Indian Plasmodium falciparum populations. Malar J. 2007;6:154.

37. Rajesh V, Singamsetti VK, Vidya S, Gowrishankar M, Elamaran M, Tripathi J, et al. Plasmodium falciparum: genetic polymorphism in apical membrane antigen-1 gene from Indian isolates. Exp Parasitol. 2008;119:144-51.

38. Farooq U, Malla N, Dubey ML. Genetic polymorphism in msp-2, ama-1 and csp genes in Plasmodium falciparum field isolates from north and north-western India. J Vector Borne Dis. 2009;46:109-16.

39. Basu M, Maji AK, Mitra M, Sengupta S. Natural selection and population genetic structure of domain-I of Plasmodium falciparum apical membrane antigen-1 in India. Infect Genet Evol. 2013;18:247-56.

40. Snounou G, Viriyakosol S, Zhu XP, Jarra W, Pinheiro L, do Rosario VE, et al. High sensitivity of detection of human malaria parasites by the use of nested polymerase chain reaction. Mol Biochem Parasitol. 1993;61:315-20.

41. Soulama I, Bigoga JD, Ndiaye M, Bougouma EC, Quagraine J, Casimiro PN, et al. Genetic diversity of polymorphic vaccine candidate antigens (apical membrane antigen-1, merozoite surface protein-3, and erythrocyte binding antigen-175) in Plasmodium falciparum isolates from western and central Africa. Am J Trop Med Hyg. 2011;84:276-84.

42. Njimoh D, Moyeh Nyuylam M, Meulah B, Bangwen D, Vakam C. Polymorphism and allelic variation of domain I of the Plasmodium falciparum apical membrane antigen-1 (ama1) gene and status of Plasmodium vivax infections in parasitized isolates from Buea, Cameroon. Issues Biol Sci Pharm Res. 2018:6:8-16.

43. Hall TA. BioEdit: a user-friendly biological sequence alignment editor and analysis program for Windows 95/98/NT. Nucleic Acids Symposium Series. 1999;41:95-8.

44. Thompson JD, Higgins DG, Gibson TJ. CLUSTAL W: improving the sensitivity of progressive multiple sequence alignment through sequence weighting, position-specific gap penalties and weight matrix choice. Nucleic Acids Res. 1994;22:4673-80.

45. Rozas J, Ferrer-Mata A, Sanchez-Del Barrio JC, Guirao-Rico S, Librado P, Ramos-Onsins SE, et al. DnaSP 6: DNA sequence polymorphism analysis of large data sets. Mol Biol Evol. 2017;34:3299-302.

46. Excoffier L, Lischer HEL. Arlequin suite ver 3.5: a new series of programs to perform population genetics analyses under Linux and Windows. Mol Ecol Resour. 2010;10:564-7.

47. Korber B. HIV signature and sequence variation analysis. In: Rodrigo AG, Learn GH, editors. Computational analysis of HIV molecular sequences. Dordrecht: Kluwer Acad Publ; 2000. p. 55-72.

48. Leigh JW, Bryant D. popart: full-feature software for haplotype network construction. Methods Ecol Evol. 2015;6:1110-6.

49. Mohapatra P, Prakash A, Bhattacharyya D, Mahanta J. Malaria situation in north-eastern region of India. ICMR Bull. 1998;28:21-30.

50. Sarma DK, Mohapatra PK, Bhattacharyya DR, Chellappan S, Karuppusamy B, Barman K, et al. Malaria in North-East India: importance and implications in the era of elimination. Microorganisms. 2019;7:673.

51. Ndiaye M, Faye B, Tine R, Ndiaye JL, Sylla K. Genetic analysis of erythrocyte binding antigen 175 (EBA-175), apical membrane antigen (AMA-1) and merozoite surface protein 3 (MSP-3) allelic types in Plasmodium falciparum isolates from rural area in Senegal. Malar Chemother Control Elim. 2014;3:2.

52. Zhu X, Zhao Z, Feng Y, Li P, Liu F, Liu J, et al. Genetic diversity of the Plasmodium falciparum apical membrane antigen I gene in parasite population from the China-Myanmar border area. Infect Genet Evol. 2016;39:155-62.

53. Kang JM, Lee J, Moe M, Jun H, Le HG, Kim TI, et al. Population genetic structure and natural selection of Plasmodium falciparum apical membrane antigen-1 in Myanmar isolates. Malar J. 2018;17:71.

54. Lumkul L, Sawaswong V, Simpalipan P, Kaewthamasorn M, Harnyuttanakorn P, Pattaradilokrat S. Unraveling haplotype diversity of the apical membrane antigen-1 gene in Plasmodium falciparum populations in Thailand. Korean J Parasitol. 2018;56:153-65.

55. Duan J, Mu J, Thera MA, Joy D, Kosakovsky Pond SL, Diemert D, et al. Population structure of the genes encoding the polymorphic Plasmodium falciparum apical membrane antigen 1: implications for vaccine design. Proc Natl Acad Sci USA. 2008;105:7857-62.
56. Wang $Y-N$, Lin M, Liang X-Y, Chen J-T, Xie D-D, Wang Y-L, et al. Natural selection and genetic diversity of domain I of Plasmodium falciparum apical membrane antigen-1 on Bioko Island. Malar J. 2019;18:317.

57. Afridi SG, Irfan M, Ahmad H, Aslam M, Nawaz M, Ilyas M, et al. Population genetic structure of domain I of apical membrane antigen-1 in Plasmodium falciparum isolates from Hazara division of Pakistan. Malar J. 2018;17:389.

\section{Publisher's Note}

Springer Nature remains neutral with regard to jurisdictional claims in published maps and institutional affiliations.
Ready to submit your research? Choose BMC and benefit from:

- fast, convenient online submission

- thorough peer review by experienced researchers in your field

- rapid publication on acceptance

- support for research data, including large and complex data types

- gold Open Access which fosters wider collaboration and increased citations

- maximum visibility for your research: over $100 \mathrm{M}$ website views per year

At BMC, research is always in progress.

Learn more biomedcentral.com/submissions 